\title{
openheart Adults with repaired tetralogy: low mortality but high morbidity up to middle age
}

\author{
Mark Dennis, ${ }^{1,2}$ Ben Moore,${ }^{2}$ Irina Kotchetkova, ${ }^{2}$ Lynne Pressley, ${ }^{2}$ \\ Rachael Cordina,,$^{1,2}$ David S Celermajer ${ }^{1,2}$
}

\begin{abstract}
To cite: Dennis M, Moore B Kotchetkova I, et al. Adults with repaired tetralogy: low mortality but high morbidity up to middle age. Open Heart 2017;4:e000564. doi:10.1136/ openhrt-2016-000564
\end{abstract}

- Prepublication history and additional material is available. To view please visit the journal (http://dx.doi.org/10.1136/ openhrt-2016-000564).

Received 10 November 2016 Accepted 22 December 2016

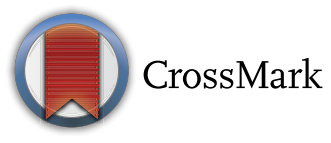

'Sydney Medical School, University of Sydney, Sydney, New South Wales, Australia 2Department of Cardiology, Royal Prince Alfred Hospital, Sydney, New South Wales, Australia

Correspondence to Professor David S Celermajer; david.celermajer@sydney. edu.au

\begin{abstract}
Objective Survival of patients with repaired tetralogy of Fallot (rToF) into young adulthood is very good. Concerns exist, however, over long-term morbidity and mortality as these subjects reach middle age. We aimed to assess survival and the prevalence of complications in patients with $\mathrm{rToF}$ seen in our Adult Congenital Heart Disease (ACHD) service.
\end{abstract}

Methods One hundred and sixty-eight consecutive patients with 'simple rToF', aged over 16 years, followed up at our tertiary-level ACHD service in Sydney, Australia since 2000, were included. We documented mortality and analysed the prospectively defined composite end points of (a) 'Serious adverse events', including death, heart failure hospitalisation and/or documented ventricular arrhythmia and (b) 'Adverse events' inclusive of the above and endocarditis, atrial arrhythmia, defibrillator and/or pacemaker implantation.

Results Mean age at the last follow-up was $34 \pm 12$ years, and $55 \%$ were men. There were $10(6 \%)$ deaths, and 26 patients (16\%) experienced a 'serious adverse event'. Fifty-one patients (30\%) experienced an 'adverse event' and 29 patients had atrial arrhythmias. One hundred and one $(61 \%)$ patients had at least one pulmonary valve replacement. By age 40 years, $93 \%$ were free of serious adverse events, and $83 \%$ were free of any adverse event. By age 50 years, only $56 \%$ had not had an adverse event. Older age and history of atrial arrhythmia were predictive of serious adverse events.

Conclusion Survival into mid-adulthood in patients with rToF is very good; however, a substantial number of survivors have adverse events by the age of 50 years.

\section{INTRODUCTION}

Improved survival of patients with congenital heart disease now means that there are more adults than children with repaired congenital disease in developed countries. Medium-term survival of repaired tetralogy of Fallot (rToF) has been documented to be good, with 20-year survival rates at or above $90 \% .^{1-6}$ This survival has resulted in a large and expanding cohort of adults with rToF.

Despite this, survival beyond the third and fourth decades is substantially lower than the age-matched normal population and is often associated with significant sequelae, including

\section{KEY QUESTIONS}

What is already known about the subject?

- Survival of patients with repaired tetralogy of Fallot throughout childhood and early adulthood is well documented. Improved survival outcomes have meant there is an expanding cohort of adult patients with repaired tetralogy of Fallot. Few previous studies have documented survival characteristics and morbidity of patients with adult congenital heart disease as they head towards middle age. The survival and any associated morbidity of these patients need to be further characterised to inform cardiologists and patients alike.

What does this study add?

- This study provides information on survival and morbidity from a large single-centre cohort of adults with repaired tetralogy of Fallot. It adds to the existing literature that although survival may be considered reasonable (above $90 \%$ ), significant morbidity develops as patients reach 50 years of age despite contemporary surgical and medical management. Furthermore, it documents atrial arrhythmias as a negative prognostic marker in these patients.

How might this impact on clinical practice?

- As patients with repaired tetralogy of Fallot move towards middle age, increased surveillance by specialist adult congenital heart disease clinics is warranted to minimise the impacts of undiagnosed and/or untreated complications.

progressive exercise intolerance, arrhythmia, right heart failure and sudden death. ${ }^{27}$

We have previously reported better functional outcomes for patients with rToF from our tertiary referral centre in Sydney, compared with elsewhere. ${ }^{8}$ Follow-up of these patients into adult life might provide important information concerning outcomes in the contemporary era. Furthermore, near-complete follow-up of these patients with adult congenital heart disease (ACHD) is facilitated by relatively low population mobility in Australia, few local ACHD 
Table 1 Basic clinical and surgical characteristics

\begin{tabular}{|c|c|c|c|c|}
\hline Variable & $\begin{array}{l}\text { All patients } \\
(n=168)\end{array}$ & $\begin{array}{l}\text { Serious adverse event } \\
\text { outcome }(n=26)\end{array}$ & $\begin{array}{l}\text { No such outcome } \\
(n=142)\end{array}$ & p Value \\
\hline Mean age at repair & $5.2 \pm 8.8$ & $9.7 \pm 10.8$ & $4.5 \pm 8.3$ & 0.01 \\
\hline Mean age at last follow-up visit & $34.1 \pm 11.7$ & $47.4 \pm 14.3$ & $31.7 \pm 9.3$ & $<0.001$ \\
\hline Gender, male & $92(54.8)$ & $20(76.9)$ & $72(50.7)$ & 0.02 \\
\hline Prior palliative shunt & $54(32.1)$ & $9(34.6)$ & $45(31.6)$ & 0.43 \\
\hline \multicolumn{5}{|l|}{ Repair details } \\
\hline Patch repair & $79(72.4)$ & $9(34.6)$ & $70(49.3)$ & 0.77 \\
\hline Conduit repair & $26(23.9)$ & $2(7.7)$ & $24(16.9)$ & \\
\hline Redo surgery required & $27(16.1)$ & $5(19.2)$ & $22(15.4)$ & 0.26 \\
\hline PVR & $101(60.1)$ & $14(54)$ & $87(61)$ & 0.66 \\
\hline Mean age at PVR, years & $25.6 \pm 12$ & $37.3 \pm 18.3$ & $23.8 \pm 9.6$ & 0.02 \\
\hline Second PVR required & $10(6.0)$ & $2(7.7)$ & $8(5.6)$ & 0.63 \\
\hline Mean age at second PVR, years & $26.4 \pm 6.9$ & $22.0 \pm 4.2$ & $27.5 \pm 7.2$ & 0.34 \\
\hline
\end{tabular}

PVR, pulmonary valve replacement.

centres and a National Death Index (NDI) that allows for complete vital status.

\section{METHODS}

\section{Study patients}

A single-centre study was performed at a tertiary-level ACHD referral centre in Sydney, Australia. This is the only ACHD centre state-wide with a referral "catchment area' of 6-7 million people.

Consecutive patients with repaired 'simple' tetralogy of Fallot (ToF) were included if they were $\geq 16$ and had been seen at least twice since January 2000 . This was a retrospective review of prospectively collected data. Patients with other congenital defects or with more complex variants of ToF, including pulmonary atresia with ventricular septal defect (PA VSD), double outlet right ventricle (RV) and/ or endocardial cushion defects, were excluded.

\section{Survival, serious adverse events and adverse event outcomes}

Survival data were obtained from the last clinic visit and cross-referenced with NDI data; thus, the vital status of all patients was obtained. Cause-of-death information was reviewed using medical record information, death certificates and autopsy information. A composite end point of 'serious adverse events' was prospectively defined as all-cause mortality, heart transplant, documented ventricular arrhythmias and/or heart failure requiring hospitalisation.

'Adverse events' were prospectively defined as the above and/or endocarditis, documented atrial arrhythmia and/or a need for permanent pacemaker or defibrillator implantation. Pulmonary valve replacement (PVR) was not included as an adverse event but, rather, as an inevitable consequence of early transannular patch-type repair of tetralogy.

A patient was classified as having an arrhythmia only if this was documented on an ECG or on interrogation of an implantable device or if there were symptoms suggestive of an arrhythmia and subsequent electrophysiological testing with a clinically relevant inducible arrhythmia.

\section{Clinical assessment}

Our centre follows standard ACHD protocols as recommended by existing guidelines. ${ }^{9}$ Patients with ToF are reviewed every 1-2 years, depending on symptoms and imaging data. Initial surgical repair reports are retrieved on all patients, if possible. Each clinic visit usually includes assessment of New York Heart Association (NYHA) class, ECG and echocardiography. Additional imaging with cardiac MRI (cMRI) was performed, as required. Available exercise stress tests, Holter monitoring, electrophysiology and cardiopulmonary exercise testing (CPET) were also reviewed.

\section{Statistical analysis}

Statistical analysis was completed using Statistical Package for Social Services V.22.0 (SPSS, Chicago Illinois). Continuous variables are presented as mean $\pm \mathrm{SD}$ or median with range. Student's unpaired t-test and Wilcoxon rank-sum test were used for comparison of continuous variables. Categorical variables are presented as frequencies and percentages. Comparison of categorical variable groups was performed using a ${ }^{2}$ test or Fischer's exact test, where applicable. Time-to-event data were assessed using Kaplan-Meier curves; analysis was capped when 20 patients or fewer were remaining for analysis. Univariate and multivariate analyses to identify predictors of predefined events were completed using logistic regression analysis. A two-tailed value of $\mathrm{p}<0.05$ was considered statistically significant.

\section{RESULTS}

The total cohort of rToF screened was 320 subjects. After exclusion of 'complicated' (defined above) rToF and patients with only one clinic visit, 168 consecutive patients aged $\geq 16$ years and with at least two clinic visits after January 2000 were included. Baseline clinical and 


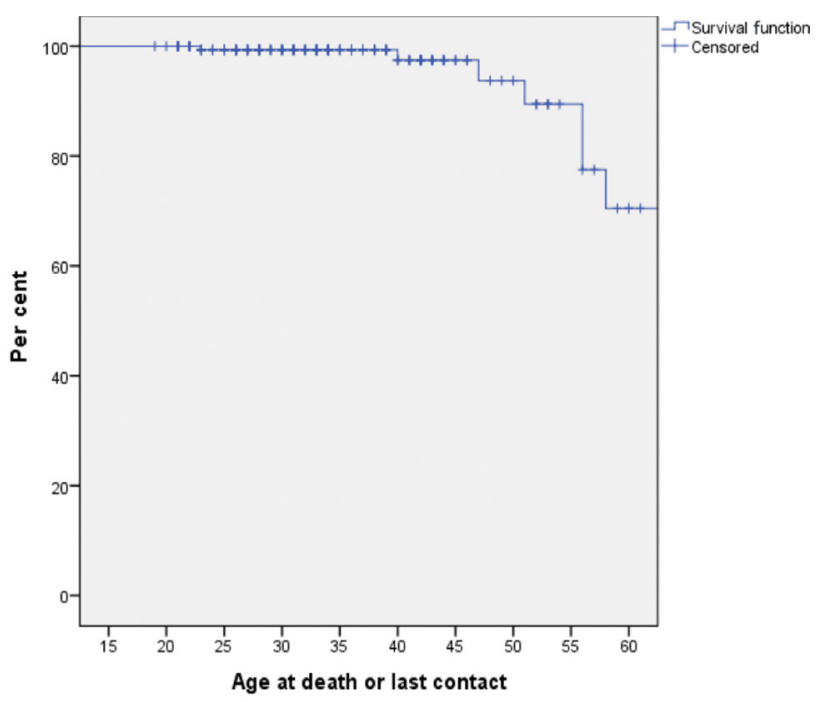

\begin{tabular}{|l|c|c|c|c|c|}
\hline Age & $\mathbf{2 0}$ & $\mathbf{3 0}$ & $\mathbf{4 0}$ & $\mathbf{5 0}$ & $\mathbf{6 0}$ \\
\hline Patients at risk & 167 & 108 & 53 & 23 & 9 \\
\hline
\end{tabular}

Figure 1 Long-term overall survival.

surgical characteristics are shown in table 1. Ninety-two $(55 \%)$ were men, and the mean follow-up period was $8 \pm 6$ years.

\section{Operative details and reinterventions}

The date of corrective surgery ranged from 1962 to 2013. Surgical repair data were available on 109 patients $(65 \%)$. The age of initial surgery was known for 159 (95\%) patients. The mean age of initial surgical repair was $5 \pm 9$ years, with a mean time period from repair to last follow-up of $29 \pm 10$ years. Fifty-four $(32 \%)$ patients had received a palliative shunt prior to complete repair. The most common repair procedure was transannular patch in 79 patients (72\%). Twenty-seven patients (16\%) required additional surgical intervention, excluding PVR.

PVR was required in 101 patients $(61 \%)$, at a mean age of $26 \pm 12$ years. A second PVR was required in $10(6 \%)$ of these patients $14 \pm 9$ years later.

\section{Survival}

The overall survival rate was $94 \%(\mathrm{n}=158)$ (figure 1). A total of 10 deaths occurred at age $57 \pm 10$ years. The most common cause of death was cardiac failure (4 deaths; $40 \%$ ). There were two deaths of unknown cause, two sudden cardiac deaths, one postoperative death and one death from breast cancer. Of those with sudden cardiac death, one had no prior history of any adverse events; the other had a history of atrial arrhythmias.

\section{Serious adverse events and adverse-event-free survival}

The composite serious adverse event outcome of mortality (10 patients), ventricular arrhythmia (12 patients), heart transplantation (none) or heart failure hospitalisation (11 patients) occurred in $26(16 \%)$ patients.

Those who suffered a serious adverse event were significantly older (mean age $47 \pm 14.3$ years, $\mathrm{p}<0.001$ ), had an older age at repair $(\mathrm{p}=0.01)$, an older age of PVR
$(37.3 \pm 18.3$ years, $\mathrm{p}<0.001)$ and were older at the last follow-up or death $(\mathrm{p}<0.001)$ compared with those without such events. Twenty-two per cent of the male patients experienced a serious adverse event compared with only $8 \%$ of female patients $(\mathrm{p}<0.001)$. Eight patients $(5 \%)$ had endocarditis. A total of $51(30 \%)$ patients experienced any adverse event at a mean age of $44 \pm 13.2$ years.

Up to 40 years of age, $93 \%$ were free from a serious adverse event, and $83 \%$ were free from any adverse event (figure 2A). By age 50 years, only $56 \%$ of the patients were free of any adverse events (figure 2B).

\section{Echocardiographic data}

Transthoracic echocardiography was completed in 164 $(98 \%)$ patients on initial visit (see online supplementary table 1). On the initial clinic visit, 26 (16\%) patients had either moderate or severe right ventricular outflow tract (RVOT) or pulmonary valve obstruction, 9 (6\%) patients had either moderate or severe RV dysfunction, and 100 (61\%) patients had either moderate or severe RV dilatation. No patient had moderate or severe left ventricular impairment on the initial or most recent review. Nine per cent of patients with no or mild RV dilatation experienced an adverse event compared with 19\% with moderate or severe $\mathrm{RV}$ dilatation $(\mathrm{p}=0.12)$. On the most recent visit, $111(68 \%)$ patients had normal RV function, and only $6(4 \%)$ patients had moderate or greater RV dysfunction. On the first echocardiogram, $12 \%$ of those with no or mild RV dysfunction suffered an adverse event compared with $67 \%$ of those with moderate or severe RV dysfunction $(\mathrm{p}<0.001)$. Moderate and severe pulmonary regurgitation was present in $93(57 \%)$ patients on the initial visit and in $49(31 \%)$ patients on the final visit.

\section{Electrophysiology}

On the most recent visit, 156 (94\%) patients were in sinus rhythm (see online supplementary table 2). Twenty-nine (17\%) patients had at least one episode of an atrial arrhythmia. Atrial flutter (eight patients) was the most common atrial arrhythmia, and 12 patients had multiple atrial arrhythmias. Fifty-two per cent of patients with a history of atrial arrhythmias suffered a serious adverse event compared with 7\% without atrial arrhythmia history $(\mathrm{p}<0.001)$. Furthermore, a larger proportion of those with a history of atrial arrhythmia experienced a ventricular arrhythmia than those with no history of atrial arrhythmia (28\% vs $3 \%, \mathrm{p}<0.001)$. Patients with moderate or severe RV dysfunction on the initial visit had a higher prevalence of atrial arrhythmias $(p<0.001)$, whereas those with moderate or severe RV dilatation on the initial visit $(\mathrm{p}=0.80)$ or previous PVR $(\mathrm{p}=0.62)$ did not.

Twelve $(7 \%)$ patients had a documented ventricular arrhythmia; of these, eight also had recorded atrial arrhythmias.

Eighteen patients $(11 \%)$ patients had electrophysiological study with or without ablation, and $13(73 \%)$ of these were indicated for atrial arrhythmias. Ten 


\section{A Serious adverse events}

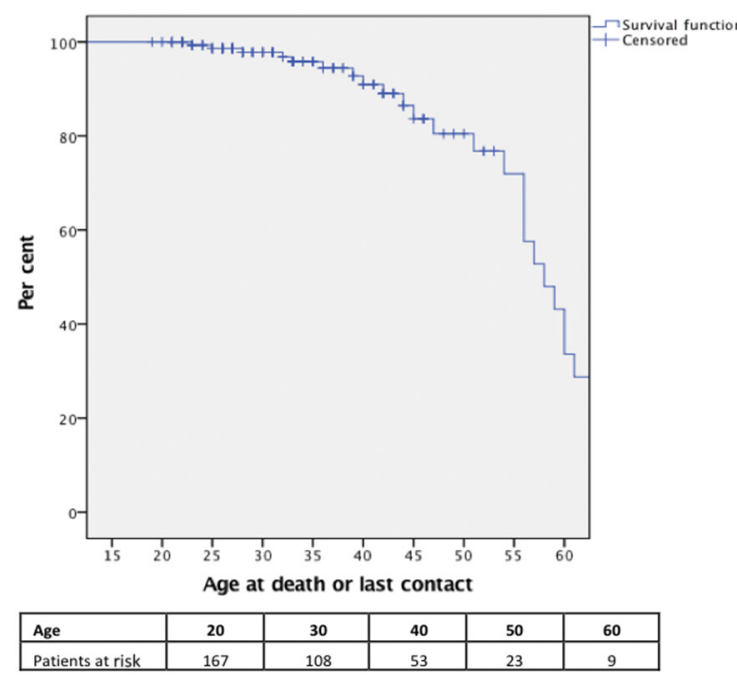

B All adverse events

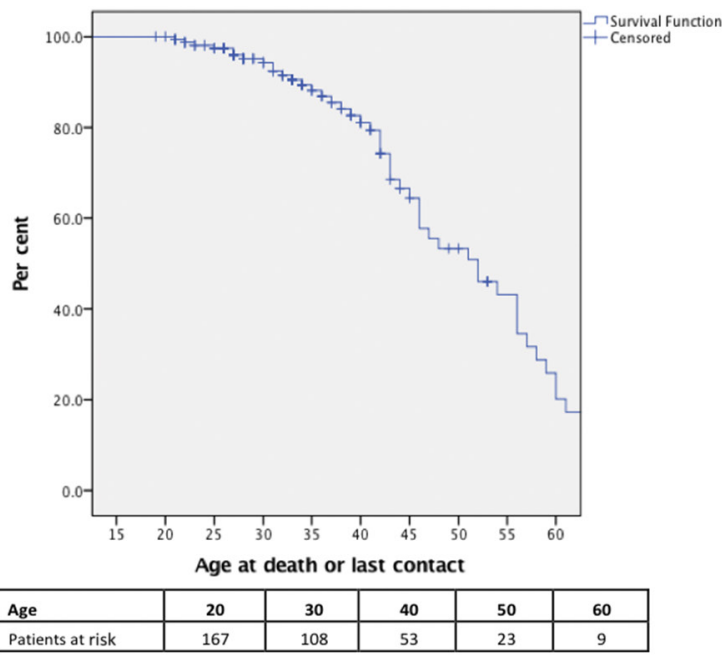

Figure 2 Age-related cumulative incidence for (A) serious adverse events (including death, heart failure admission, ventricular arrhythmia and cardiac transplant) and (B) all adverse events (including serious adverse event and endocarditis, atrial arrhythmia, defibrillator and pacemaker implantation).

(5\%) patients required a permanent pacemaker, with a mean age of implantation of $28 \pm 24$ years, usually for second-degree or third-degree atrioventricular block in six $(67 \%)$ patients. Fifteen $(9 \%)$ patients had a defibrillator implanted at a mean age of $37 \pm 9$ years; 12 of these were for secondary prevention. Five patients had device therapy, and one of these required defibrillation for ventricular tachycardia (VT). Four patients had experienced inappropriate shocks from their device, and all of these were secondary prevention devices.

\section{Heart failure status}

Sixteen (10\%) patients were on at least one cardiac failure medication on the initial consultation and 13 $(8 \%)$ on their most recent visit. Eleven $(7 \%)$ patients required hospitalisation during their follow-up period. No patients were listed for heart transplant.

The NYHA status was available on 163 (97\%) patients, and $158(94 \%)$ patients were class I at the last follow-up.

\section{Predictors of mortality and serious adverse events and mortality}

A higher proportion of those who died had experienced atrial arrhythmias than those who were alive $(63 \%$ vs $15 \%, \mathrm{p}=0.005)$. When logistic regression analysis was performed and corrected for age, this was not a significant predictor (OR 2.53, CI 0.41 to 15.5).

On univariate analysis, older age (OR 1.1, 95\% CI 1.07 to 1.17 ), male gender (OR $3.2,95 \%$ CI 1.23 to 8.55 ), moderate-to-severe RV dysfunction on initial visit (OR 14.3, 95\% CI 3.3 to 62.1 ), history of atrial arrhythmia (OR 15.2, CI 5.64 to 41.2 ) and age at repair (OR 1.04, CI 0.01 to 1.09) were statistically significantly with regard to late serious adverse events (table 2). On multivariate analysis, only older age and a history of atrial arrhythmia remained as significant predictors for serious adverse events.

\section{DISCUSSION}

This single-centre contemporary study of 168 patients with 'simple' rToF identified that despite very good survival into young adulthood, a substantial proportion of patients with rToF develop significant morbidity on entering middle age.

The overall mortality of the cohort (mean age of $34 \pm 12$ years and a mean time from initial surgery of $29 \pm 10$ years) was $6 \%$, with a median age at death of 57 years. This survival is comparable to other studies, with long-term (>30 years) survival rates at or above $90 \% .^{2}$ 4-6 10-12 However, survival into adulthood is still substantially lower than for the age-matched population. ${ }^{56}$

\begin{tabular}{lllll} 
Table 2 & Univariate and multivariate predictors of serious adverse events \\
\hline \multirow{2}{*}{ Variable } & Univariate model & \multicolumn{3}{l}{ Multivariate model II* } \\
\cline { 2 - 5 } & OR $\mathbf{( 9 5 \% ~ C l )}$ & p Value & OR (95\% Cl) & p Value \\
\hline Age & $1.12(1.07$ to 1.17$)$ & $<0.0001$ & $1.08(1.03$ to 1.13$)$ & 0.003 \\
Sex & $3.24(1.23$ to 8.55$)$ & 0.017 & $3.46(0.98$ to 12.2$)$ & 0.054 \\
Right ventricular dysfunction on the first visit & $14.32(3.3$ to 62.1$)$ & $<0.0001$ & $5.32(0.95$ to 29.9$)$ & 0.058 \\
History of atrial arrhythmia & $15.24(5.64$ to 41.16$)$ & $<0.0001$ & $5.03(1.49$ to 16.9$)$ & 0.009 \\
\hline
\end{tabular}

${ }^{*}$ Excluding the age of repair. 
In our series, all deaths occurred after the age of 40 years. The most common causes of death were heart failure and sudden cardiac death, in keeping with other studies. ${ }^{13}{ }^{14}$ Sudden cardiac death is well documented in the rToF population with variable reported risk factors, many thought to relate to chronic RV volume overload over time.

Our data indicate that despite reasonable survival to middle age, significant morbidity occurs. We chose a composite end point for serious adverse events of mortality, ventricular arrhythmia and/or heart failure requiring hospitalisation due to their clinical relevance and likely prognostic importance. Serious-event-free survival declined markedly after the age of 40 years. Of patients aged 40 years, $93 \%$ were free from serious adverse events. This declined to $82 \%$ for those above 50 years of age. Other studies report a cumulative-event-free survival (reinterventions, PVR, arrhythmias, heart failure, stroke and endocarditis) at 40-year follow-up of only $25 \% .^{2}$ As PVR is an expected outcome after transannular patch repairs, we excluded this as an 'adverse event', accounting for some of the differences with previous reports. When we included other adverse events (other than PVR), only $56 \%$ of the patients were from any adverse event by 50 years of age.

We found that male gender, moderate-to-severe RV dysfunction on the initial clinic visit, and history of atrial arrhythmias were predictors of serious adverse events on univariate analysis. On multivariate analysis, only a history of atrial arrhythmias and age remained significant predictors. As opposed to other studies, ${ }^{1-36}$ patch-based repair, presence of a palliative shunt, RV dilatation and presence of severe pulmonary regurgitation were not found to be predictive of serious adverse outcomes. We have previously shown preserved exercise capacity and stroke volume even with severe RV dilation and significant pulmonary regurgitation; ${ }^{8}$ however, RV dysfunction has been a more consistent predictor of adverse events in previous studies.

The prevalence of atrial arrhythmias in our study was $17 \%$. Other patient cohorts varied widely in their prevalence $(2.5 \%-54 \%) \cdot{ }^{12}{ }^{15-17}$ In a study by Gatzoulis $e t a l,{ }^{3}$ only $29(3.7 \%)$ of 793 patients exhibited sustained atrial fibrillation or flutter. This cohort had an older mean age of repair ( $8.2 \pm 8$ years), did exclude PA VSDs, but were significantly younger than our population at the last follow-up, with a mean age of 27.5 years. Older cohorts ${ }^{216}$ had higher cumulative incidence of arrhythmias, and older age is a known predictor of the occurrence of atrial arrhythmias. Atrial arrhythmias in patients with $\mathrm{rToF}$ are not a benign pathology and are associated with increased adverse events, including later development of congestive cardiac failure, reoperation, ventricular dysfunction and arrhythmias, stroke and death. ${ }^{1017}$ Our data support atrial arrhythmias as a poor prognostic sign. Abnormal right atrial size ${ }^{19}$ and function ${ }^{20}$ exist in rToF and have been shown to strongly predict sustained tachyarrhythmia and clinical outcomes but were not formally assessed in our study.
Ventricular arrhythmia is a well-documented complication in rToF and occurred in $12(7 \%)$ of our patients. In a previous cross-sectional study of 566 patients, ${ }^{16}$ the prevalence of ventricular arrhythmias was $15 \%$; however, this is higher than other series. ${ }^{3}$ Recently, Valente $e t a l^{10}$ reported only 4 patients (out of 873) with documented sustained VT (median age of 29.3 years). The incidence of ventricular arrhythmias, such as atrial arrhythmias, has been shown to increase with time from repair and with older age, especially after the age of 45 years, ${ }^{16}$ and this may, in part, explain our higher prevalence of ventricular arrhythmias than in some cohorts. Although our study did not show RV dilatation as a predictor of adverse events, its association with QRS widening and adverse events has been well documented. ${ }^{311} 16$

Reintervention postrepair is also a concern in the longterm follow-up of patients with rToF. Twenty-seven (16\%) patients required a second surgical procedure other than PVR, with a majority of these occurring before the age of 16. Sixty-one per cent of the patients in our cohort required a PVR at a mean age of 26 years. Previous studies ${ }^{24}$ had reintervention rates of $40 \%-50 \%$, inclusive of PVR. Fifty per cent to eighty per cent ${ }^{21} 22$ of patients with PVR may exhibit valve dysfunction or failure at 10 years; it is thus very likely that many patients will require a second or third PVR in their lifetime. With an early operative mortality of $1 \%-2 \%,{ }^{23}$ multiple PVRs represent a significant cumulative risk to patients.

Ventricular dysfunction and the development of heart failure occur over time in patients with rToF. Our study showed that while RV dilatation is common, significant RV dysfunction occurred in a small number of patients. This is significantly lesser than the results reported by Cuypers et $a l^{2}{ }^{2}$ who previously showed impaired RV dysfunction in $>75 \%$ and left ventricular functional impairment in $50 \%$ of patients at 40 years' follow-up, many with ventricular diastolic dysfunction also. Nonetheless, a significant proportion of deaths in our study and other contemporary cohorts ${ }^{10}$ are due to heart failure, and traditional heart failure strategies may not produce the same benefit seen in the non-congenital heart failure population.

In our study, only older age and a history of atrial arrhythmias were associated with serious adverse events. While ventricular arrhythmias have been clearly associated with RV dilatation, increased QRS width and sudden death, ${ }^{34}$ the mechanism linking atrial arrhythmias to adverse events is less clear.

\section{Study limitations}

As an ACHD service, only patients over the age of 16 years are seen, and as such, there is 'attrition' from paediatric services. In addition, being a referral service, there is a likelihood of referral bias. Furthermore, despite best efforts, full operation reports were not available in 59 $(35 \%)$ patients, limiting analysis and conclusions that can be drawn regarding the type of initial surgical repair. Geographical movement of patients over time does complicate follow-up; however, being the only ACHD 
clinic in New South Wales, this effect is minimised, and use of the NDI ensures near-complete follow-up for vital status.

Similar to other long-term follow-up studies, changes in surgical practices over time is likely to produce different subgroup results. Formal QRS measurements were not available on all patients. Although all available cMRI data were reviewed, not enough of the cohort had serial cMRI data to warrant separate analyses. We recognise that assessment of RV anatomy using echocardiography is difficult and the analyses was not blinded. As patients age, traditional cardiovascular risk factors, including diabetes mellitus, hypertension and coronary disease, become increasingly important. These data, although collected, were not in easily extractable form. Finally, there are limited patients at risk above the age of 50 years, limiting the power of statistical analysis in this group of patients.

\section{CONCLUSION}

Our study shows that while survival of patients with rToF into adulthood is very good, the transition into middle age is associated with significant morbidity and increased risk of mortality. Given the marked increase in events from middle age, careful and expert surveillance is warranted.

\section{Competing interests None declared.}

Provenance and peer review Not commissioned; internally peer reviewed.

Data sharing statement No additional unpublished data from this study is currently available. Any requests for data sharing should be made to the corresponding author.

Open Access This is an Open Access article distributed in accordance with the Creative Commons Attribution Non Commercial (CC BY-NC 4.0) license, which permits others to distribute, remix, adapt, build upon this work non-commercially, and license their derivative works on different terms, provided the original work is properly cited and the use is non-commercial. See: http://creativecommons.org/ licenses/by-nc/4.0

C Article author(s) (or their employer(s) unless otherwise stated in the text of the article) 2017. All rights reserved. No commercial use is permitted unless otherwise expressly granted.

\section{REFERENCES}

1. Chiu SN, Wang JK, Chen HC, et al. Long-term survival and unnatural deaths of patients with repaired Tetralogy of Fallot in an Asian cohort. Circ Cardiovasc Qual Outcomes 2012;5:120-5.

2. Cuypers JA, Menting ME, Konings EE, et al. Unnatural history of tetralogy of Fallot: prospective follow-up of 40 years after surgical correction. Circulation 2014;130:1944-53.

3. Gatzoulis MA, Balaji S, Webber SA, et al. Risk factors for arrhythmia and sudden cardiac death late after repair of Tetralogy of Fallot: a multicentre study. Lancet 2000;356:975-81.

4. Hickey EJ, Veldtman G, Bradley TJ, et al. Late risk of outcomes for adults with repaired Tetralogy of Fallot from an inception cohort spanning four decades. Eur J Cardiothorac Surg 2009;35:156-64.
5. Murphy JG, Gersh BJ, Mair DD, et al. Long-term outcome in patients undergoing surgical repair of Tetralogy of Fallot. N Engl J Med 1993;329:593-9.

6. Nollert G, Fischlein T, Bouterwek S, et al. Long-term results of total repair of Tetralogy of Fallot in adulthood: 35 years follow-up in 104 patients corrected at the age of 18 or older. Thorac Cardiovasc Surg 1997:45:178-81.

7. Apitz C, Webb GD, Redington AN. Tetralogy of Fallot. Lancet 2009;374:1462-71.

8. O'Meagher S, Munoz PA, Alison JA, et al. Exercise capacity and stroke volume are preserved late after tetralogy repair, despite severe right ventricular dilatation. Heart 2012;98:1595-9.

9. Bhatt $\mathrm{AB}$, Foster $\mathrm{E}, \mathrm{Kuehl} \mathrm{K}$, et al; American Heart Association Council on Clinical Cardiology. Congenital heart disease in the older adult: a scientific statement from the American Heart Association. Circulation 2015;131:1884-931.

10. Valente AM, Gauvreau K, Assenza GE, et al. Contemporary predictors of death and sustained ventricular tachycardia in patients with repaired Tetralogy of Fallot enrolled in the INDICATOR cohort. Heart 2014:100:247-53.

11. Knauth AL, Gauvreau K, Powell AJ, et al. Ventricular size and function assessed by cardiac MRI predict major adverse clinical outcomes late after tetralogy of Fallot repair. Heart 2008;94:211-6.

12. Nakazawa M, Shinohara T, Sasaki A, et al; Study Group for Arrhythmias Long-Term After Surgery for Congenital Heart Disease: ALTAS-CHD study. Arrhythmias late after repair of Tetralogy of Fallot: a Japanese multicenter study. Circ J 2004;68:126-30.

13. Nørgaard MA, Lauridsen P, Helvind M, et al. Twenty-to-thirty-sevenyear follow-up after repair for Tetralogy of Fallot. Eur $J$ Cardiothorac Surg 1999;16:125-30.

14. Oechslin EN, Harrison DA, Connelly MS, et al. Mode of death in adults with congenital heart disease. Am J Cardiol 2000;86:1111-6.

15. Arya S, Kovach J, Singh $\mathrm{H}$, et al. Arrhythmias and sudden death among older children and young adults following Tetralogy of Fallot repair in the current era: are previously reported risk factors still applicable? Congenit Heart Dis 2014;9:407-14.

16. Khairy P, Aboulhosn J, Gurvitz MZ, et al. Alliance for Adult Research in Congenital Cardiology (AARCC). Arrhythmia burden in adults with surgically repaired Tetralogy of Fallot: a multi-institutional study. Circulation 2010;122:868-75.

17. Roos-Hesselink J, Perlroth MG, McGhie J, et al. Atrial arrhythmias in adults after repair of Tetralogy of Fallot. Correlations with clinical, exercise, and echocardiographic findings. Circulation 1995;91:2214-9.

18. Harrison DA, Siu SC, Hussain F, et al. Sustained atrial arrhythmias in adults late after repair of Tetralogy of Fallot. Am J Cardiol 2001;87:584-8.

19. Bonello B, Kempny A, Uebing A, et al. Right atrial area and right ventricular outflow tract akinetic length predict sustained tachyarrhythmia in repaired Tetralogy of Fallot. Int $J$ Cardiol 2013;168:3280-6.

20. Luijnenburg SE, Peters RE, van der Geest RJ, et al. Abnormal right atrial and right ventricular diastolic function relate to impaired clinical condition in patients operated for Tetralogy of Fallot. Int J Cardiol 2013;167:833-9.

21. Cheung EW, Wong WH, Cheung YF. Meta-analysis of pulmonary valve replacement after operative repair of Tetralogy of Fallot. Am J Cardiol 2010;106:552-7.

22. Lee $\mathrm{C}, \mathrm{Kim} \mathrm{YM}$, Lee $\mathrm{CH}$, et al. Outcomes of pulmonary valve replacement in 170 patients with chronic pulmonary regurgitation after relief of right ventricular outflow tract obstruction: implications for optimal timing of pulmonary valve replacement. J Am Coll Cardiol 2012;60:1005-14.

23. Babu-Narayan SV, Diller GP, Gheta RR, et al. Clinical outcomes of surgical pulmonary valve replacement after repair of tetralogy of Fallot and potential prognostic value of preoperative cardiopulmonary exercise testing. Circulation 2014;129:18-27.

24. Gatzoulis MA, Till JA, Somerville J, et al. Mechanoelectrical interaction in Tetralogy of Fallot. QRS prolongation relates to right ventricular size and predicts malignant ventricular arrhythmias and sudden death. Circulation 1995;92:231-7. 